\title{
Grandezas físicas e análise dimensional: da mecânica à gravidade quântica
}

\author{
Physical quantities and dimensional analysis: from mechanics to quantum gravity
}

\author{
Diego Trancanelli*
}

Instituto de Física, Universidade de São Paulo, São Paulo, SP, Brasil

Recebido em 1 de dezembro de 2015. Revisado em 30 de janeiro de 2016; Aceito em 10 de fevereiro de 2016

Entre os primeiros conceitos que alunos de graduação em física encontram nos seus estudos, há os de grandezas e dimensões físicas. Neste artigo pedagógico de revisão, usando a poderosa ferramenta da análise dimensional, vou partir desses conceitos para uma viagem através de vários ramos da física teórica, da mecânica até a gravidade quântica. Entre outras coisas, vou discutir um pouco sobre as constantes fundamentais da Natureza, o chamado "cubo da física" e o sistema de unidades naturais.

Palavras-chave: análise dimensional, constantes fundamentais, unidades naturais.

Physical quantities and physical dimensions are among the first concepts encountered by students in their undergraduate career. In this pedagogical review, I will start from these concepts and, using the powerful tool of dimensional analysis, I will embark in a journey through various branches of physics, from basic mechanics to quantum gravity. I will also discuss a little bit about the fundamental constants of Nature, the so-called "cube of Physics", and the natural system of units.

Keywords: dimensional analysis, fundamental constants, natural units.

\section{Introdução}

O conceito de grandeza física é um dos mais básicos entre os conceitos encontrados pelos alunos que começam o estudo da física, sendo, talvez, até axiomático. Grandezas físicas são usadas para descrever fenômenos ou propriedades de sistemas e são caracterizadas por terem dimensões como, por exemplo, dimensões de massa, força ou energia.

As dimensões podem ser primitivas ou derivadas. As dimensões primitivas são três: a massa (que indicamos com a letra $M$ ), o comprimento (que indicamos com a letra $L$, da palavra inglês length) e o tempo (que indicamos com a letra $T$ ). Todas as outras dimensões são derivadas, ou seja, podem ser expressas em termos das três primitivas. O leitor é convidado a abrir um livro-texto básico qualquer, como [1], e verificar isso explicitamente 1 Por exem-

\footnotetext{
*Endereço de correspondência: dtrancan@usp.br.

${ }^{1}$ Contudo, às vezes é útil considerar outras dimensões como se fossem primitivas, para motivos práticos. Por exemplo, poderíamos considerar a temperatura e a carga elétrica também
}

plo, uma aceleração tem dimensões de comprimento sobre tempo ao quadrado, $L T^{-2}$, enquanto a energia tem dimensões de massa vezes comprimento ao quadrado sobre tempo ao quadrado, $M L^{2} T^{-2}$.

No que segue, indicamos as dimensões de uma grandeza física $X$ com colchetes: $[X]$. As dimensões de $X$ são, então, dadas por uma certa combinação das dimensões primitivas

$$
[X]=M^{\alpha} L^{\beta} T^{\gamma},
$$

onde $\alpha, \beta$ e $\gamma$ são números inteiros ou racionais, positivos ou negativos ${ }^{2}$ Nos exemplos anteriores, sobre aceleração e energia, esses números são, respectivamente, $(\alpha=0, \beta=1, \gamma=-2)$ e $(\alpha=1, \beta=$

como primitivas, mas é importante ressaltar que isso não é estritamente necessário. De fato, a temperatura tem as mesmas dimensões de energia e a carga elétrica tem dimensões de $\sqrt{M L^{3} T^{-2}}$. Em outras palavras, a constante de Boltzmann $k_{B}$ e a constante de Coulomb $1 / 4 \pi \varepsilon_{0}$ são simplesmente fatores de conversão e não exercem um papel fundamental.

${ }^{2}$ A dependência de $[X]$ em $M, L$ e $T$ não pode ser mais complicada que o monômio em (1) por razões que logo ficarão claras. 
$2, \gamma=-2)$. Além das grandezas físicas dimensionais, há também os números puros, como 0,1 ou $\pi$, que são adimensionais, ou seja, têm expoentes $(\alpha=0, \beta=0, \gamma=0)$.

Às vezes, é útil mudar de base de dimensões. A nova base precisa ser composta de dimensões independentes e precisa ser completa. Por exemplo, força $(F)$, comprimento e tempo representam uma base alternativa legítima, pois $F=M L T^{-2}$ contém $M$. Por outro lado, velocidade $(V)$, comprimento e tempo não seria uma base legítima, pois $V=L T^{-1}$ não é independente das outras duas e não é possível descrever massas nessa base. Veremos na Seção 4 que existe uma base muito conveniente de dimensões, chamada justamente de base "natural".

Depois dessa breve introdução, estamos prontos para discutir o que é a análise dimensional.

\section{Análise dimensional}

A análise dimensional é a arte de antecipar como uma certa grandeza vai depender de outras grandezas presentes em um problema, sem fazer a conta detalhada, ou seja, sem ter que resolver explicitamente as equações que definem o problema. A análise dimensional é uma ferramenta extremamente poderosa na caixa de ferramentas de um físico, teórico ou experimental, e é muito útil para várias tarefas, entre elas:

- Simplificar problemas. A análise dimensional permite entender quais são as combinações de grandezas físicas relevantes para o problema abordado. Abaixo, vamos demonstrar esse ponto através de vários exemplos concretos.

- Desenvolver intuição física. Uma das habilidades mais importantes para um físico é ter intuição, ou seja, a capacidade de imaginar o comportamento de um sistema ao mudarmos os parâmetros que o controlam, sem fazer contas detalhadas. A análise dimensional é uma das técnicas mais úteis para alcançar isso.

- Detectar erros. Talvez essa seja a aplicação mais imediatamente relevante para os alunos. Uma boa parte dos erros na solução de problemas pode ser detectada simplesmente checando se as dimensões do resultado final são apropriadas para a grandeza que se pretende calcular.

O princípio básico da análise dimensional é o de homogeneidade dimensional de qualquer equação. Isso significa que todos os termos da equação têm que ter as mesmas dimensões. Enquanto podemos combinar (multiplicando ou dividindo) grandezas físicas com dimensões diferentes, somente podemos comparar (somando ou subtraindo) grandezas físicas com as mesmas dimensões. Outra maneira de falar isso, em termos mais populares, é que só podemos comparar bananas com bananas e laranjas com laranjas, e não bananas com laranjas.

O procedimento para entender como uma grandeza $Y$ depende das outras grandezas do problema usando a análise dimensional é o seguinte:

1. Começamos escrevendo as dimensões $[Y]$ de $Y$ em termos das três dimensões primitivas

$$
[Y]=M^{\alpha} L^{\beta} T^{\gamma} .
$$

Isso determina os expoentes $(\alpha, \beta, \gamma)$.

2. Tentamos identificar as grandezas $X_{i}(i=$ $1,2, \ldots)$ das quais $Y$ pode depender. Essa é a parte mais complicada do procedimento e é graças a ela que, como escrevi acima, a análise dimensional pode ser considerada uma arte! Por exemplo, será que o período de um pêndulo simples depende da temperatura da sala onde ele se encontra? Veremos vários exemplos nas próximas seções como decidir isso na prática.

3. Escrevemos $Y$ como um monômio destas grandezas $X_{i}$, com uma quantidade adimensional $C$ na frente

$$
Y=C X_{1}^{a_{1}} X_{2}^{a_{2}} \ldots,
$$

onde $a_{1}, a_{2}, \ldots$ são expoentes a serem determinados.

4. Comparamos a equação acima com (2) e resolvemos para os expoentes $a_{1}, a_{2}, \ldots$

$$
\left[X_{1}^{a_{1}}\right]\left[X_{2}^{a_{2}}\right] \ldots=M^{\alpha} L^{\beta} T^{\gamma},
$$

lembrando que $[C]=1$, pois $C$ é adimensional. Por exemplo, consideramos o caso em que haja somente três grandezas relevantes para o problema: $X_{1}, X_{2}$ e $X_{3}$. Imaginemos também que $X_{1}$ tenha dimensões dadas pela escolha de expoentes $\left(\alpha_{1}, \beta_{1}, \gamma_{1}\right), X_{2}$ tenha $\left(\alpha_{2}, \beta_{2}, \gamma_{2}\right)$ e, finalmente, $X_{3}$ tenha $\left(\alpha_{3}, \beta_{3}, \gamma_{3}\right)$. O sistema de equações a ser resolvido resulta ser

$$
\begin{gathered}
a_{1} \alpha_{1}+a_{2} \alpha_{2}+a_{3} \alpha_{3}=\alpha, \\
a_{1} \beta_{1}+a_{2} \beta_{2}+a_{3} \beta_{3}=\beta, \\
a_{1} \gamma_{1}+a_{2} \gamma_{2}+a_{3} \gamma_{3}=\gamma .
\end{gathered}
$$


Encontrando as soluções para $a_{1}, a_{2}$ e $a_{3}$ podemos, portanto, determinar a dependência de $Y$ em relação a $X_{1}, X_{2}$ e $X_{3}$.

Seguem agora três observações importantes. A primeira é sobre os argumentos de funções, como, por exemplo, funções trigonométricas ou a função exponencial. Esses argumentos têm que ser adimensionais. É fácil se convencer disso observando que na expansão destas funções, devido ao princípio da homogeneidade dimensional, todos os termos têm que ter as mesmas dimensões. Por exemplo,

$$
\cos x=1-\frac{x^{2}}{2}+\frac{x^{4}}{24}+\ldots,
$$

$\operatorname{logo} x$ deve ser adimensional, sendo o primeiro termo do lado direito da equação, 1 , adimensional.

A segunda observação se chama Teorema-П de Buckingham [2] e é uma contagem muito simples de quantas combinações adimensionais independentes podem ser formadas a partir das grandezas do problema. Se houver $n$ grandezas, o número $r$ de combinações adimensionais que pode ser obtido é dado por ${ }^{3}$

$$
r=n-3 .
$$

Chamaremos essas combinações adimensionais de $\Pi_{a}$, com $a=1, \ldots, r$. É fácil ver (e veremos, logo, em uma série de exemplos) que, para $r \leq 1$, a quantidade $C$ introduzida antes em (3) é uma constante (como, por exemplo, $2 \pi$ ou $1 / 2$ ), mas, para $r \geq 2$, a $C$ será uma função de algumas das combinações $\Pi_{a}$.

Finalmente, a última observação é que, quando tomamos limites, devemos tomar cuidado para que o limite faça sentido, ou seja, para estarmos comparando, no limite, quantidades com as mesma dimensões. Em particular, falar que $x \ll 1$ ou $x \gg 1$, somente faz sentido se $x$ for um número puro como 1. Voltaremos a esse ponto mais adiante.

Vamos testar logo essas noções em alguns exemplos simples de mecânica clássica.

\section{Exemplo 1: Período de um pêndulo}

Podemos entender como o período de um pêndulo depende dos outros parâmetros do problema usando a análise dimensional. Primeiramente, notamos que há

\footnotetext{
${ }^{3} \mathrm{O} 3$ nessa equação se deve ao fato de ter sido escolhido trabalhar com três dimensões primitivas. Incluindo, por exemplo, temperatura e carga elétrica como dimensões primitivas, além de $M, L$ e $T$, devemos substituir o 3 por um 5 .
}

cinco grandezas relevantes no problema:4 o período $\tau$ (com dimensões de $T$ ), o comprimento da corda $\ell$ (com dimensões de $L$ ), a massa suspensa $m$ (com dimensões de $M$ ), a aceleração da gravidade $g$ (com dimensões de $L T^{-2}$ ) e o ângulo $\theta_{0}$ do deslocamento inicial do pêndulo (que é adimensional) $!^{5}$

Ver a Figura 1. Conforme o teorema-П, haverá $r=5-3=2$ combinações adimensionais. A primeira combinação é, claramente, dada por $\Pi_{1}=\theta_{0}$, enquanto a segunda pode ser obtida combinando $\tau, \ell, m$ e $g$. Usando o procedimento descrito acima, podemos escrever

$$
\tau=C\left(\theta_{0}\right) \ell^{a_{1}} m^{a_{2}} g^{a_{3}},
$$

e, em termos das dimensões,

$$
\begin{aligned}
& {[\tau]=\left[C\left(\theta_{0}\right)\right][\ell]^{a_{1}}[m]^{a_{2}}[g]^{a_{3}} \rightarrow} \\
& T=L^{a_{1}+a_{3}} M^{a_{2}} T^{-2 a_{3}},
\end{aligned}
$$

que tem solução única dada por $a_{1}=\frac{1}{2}, a_{2}=0 \mathrm{e}$ $a_{3}=-\frac{1}{2}$. Logo

$$
\tau=C\left(\theta_{0}\right) \sqrt{\frac{\ell}{g}} .
$$

Essa conta simples revela que o período não pode depender da massa do pêndulo! É claro que a análise dimensional não é suficiente para determinar a função $C\left(\theta_{0}\right)$, que é a parte difícil do problema e que pode ser obtida somente resolvendo a equação diferencial do pêndulo. Se estivermos interessados na comparação entre dois pêndulos diferentes (de períodos $\tau_{1}$ e $\tau_{2}$ e comprimentos $\ell_{1}$ e $\ell_{2}$ ), mas com o mesmo

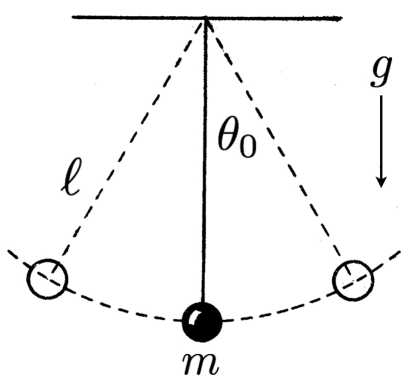

Figura 1: Pêndulo simples.

\footnotetext{
${ }^{4}$ Como mencionado antes, essa é a parte difícil da análise: entender o que é relevante e o que não é relevante (como, por exemplo, a temperatura do ambiente em volta do pêndulo). ${ }^{5}$ Ângulos precisam ser adimensionais devido à periodicidade: $\theta_{0}$ deve ser identificado com $\theta_{0}+2 \pi$, e portanto $\theta_{0}$ deve ter as mesmas dimensões de $2 \pi$.
} 
ângulo inicial, essa função é de qualquer forma irrelevante, pois

$$
\frac{\tau_{1}}{\tau_{2}}=\sqrt{\frac{\ell_{1}}{\ell_{2}}} .
$$

O leitor é convidado a repetir a mesma análise para um oscilador harmônico de massa $m$ e constante elástica $k$.

\section{Exemplo 2: Energia de uma corda vibrante}

Agora, queremos estimar a energia de uma corda de violão em vibração. As variáveis do problema são: a energia $E$, o comprimento da corda $\ell$, a amplitude das oscilações $A$ (parece claro que, quanto mais ampla é a oscilação, maior é a energia), a densidade linear de massa $\rho$ da corda (com dimensões de $M L^{-1}$ ) e a tensão $s$ da corda (com dimensões de força, $M L T^{-2}$ ). De novo há $r=5-3=2$ combinações adimensionais independentes que são $\Pi_{1}=E / A s$ e $\Pi_{2}=\ell / A$. Portanto, a energia será

$$
E=A s f(\ell / A)
$$

onde $f(\ell / A)$ é uma função (adimensional) que, claramente, não pode ser determinada usando a análise dimensional. De qualquer forma, a análise foi útil para simplificar o problema, determinando a dependência da energia com a tensão da corda.

\section{Exemplo 3: Potência gasta por um mexedor de café}

Agora consideremos uma situação do cotidiano: quanta energia gastamos para mexer o açúcar no nosso café? É claro que esse problema é extremamente complicado e depende de muitos parâmetros. A análise dimensional, contudo, permite simplificar as coisas um pouco. Podemos imaginar que as grandezas (dimensionais) mais relevantes para o problema sejam: a potência $P$ gasta (ou seja, a energia gasta por unidade de tempo), a densidade volumétrica $\rho$ do café e a sua viscosidade $\mu$, o tamanho do mexedor (que podemos imaginar cilíndrico, com diâmetro $d$ ) e, enfim, a velocidade $v$ do movimento. Os leitores são convidados a escrever as dimensões de todas essas grandezas. De novo, temos $r=5-3=2$ combinações $\Pi_{a}$ adimensionais. A primeira combinação depende das características do fluido e se chama número de Reynolds $\mathrm{R} \AA^{6}$

$$
\Pi_{1} \equiv \operatorname{Re}=\frac{\rho v d}{\mu},
$$

enquanto a segunda combinação se chama número de potência $N_{\mathrm{p}}$ e é dada por

$$
\Pi_{2} \equiv N_{\mathrm{p}}=\frac{P}{\rho v^{3} d^{5}} .
$$

Vemos, de novo, que a análise dimensional não foi suficiente para resolver o problema, como era esperado dada a complexidade do mesmo, mas, pelo menos, separou duas combinações adimensionais que serão relevantes. A potência gasta vai, de fato, depender das outras grandezas como segue

$$
P=\rho v^{3} d^{5} f(\mathrm{Re}),
$$

para uma certa função (adimensional) $f$ a ser determinada.

\section{Exemplo 4: Energia liberada por bombas atômicas}

Uma outra aplicação interessante da análise dimensional é estimar quanta energia é liberada na explosão de uma bomba atômica [3]. Na década de '50, o físico inglês G. I. Taylor estudou fotos de explosões como a da Figura 2.

Nessas fotos, podemos ler o tamanho da frente de choque da onda da explosão (um comprimento que chamamos de $R$ ) em função do tempo $t$. A observação importante é que podemos desprezar a pressão do ar em volta da explosão, enquanto somente a densidade volumétrica $\rho$ do ar (com dimensões de $M L^{-3}$ ) é importante. A energia liberada na explosão deve, portanto, ser dada por

$$
E=C \frac{\rho R^{5}}{t^{2}}
$$

sendo $C$ uma constante adimensional. Extraindo $R(t)$ das fotos é possível, portanto, conhecer $E$, a menos da constante $C$, que deve ser obtida com outros métodos.

\section{Exemplo 5: Velocidade de barcos de remos}

Finalmente, vamos tentar estimar como a velocidade de barcos de remos depende do número de remadores [4] (ver também [5]). As grandezas relevantes

\footnotetext{
${ }^{6}$ Esses números são muito importantes no estudo da dinâmica dos fluidos, em particular no estudo da turbulência, que acontece quando o fluido tem número de Reynolds grande.
} 


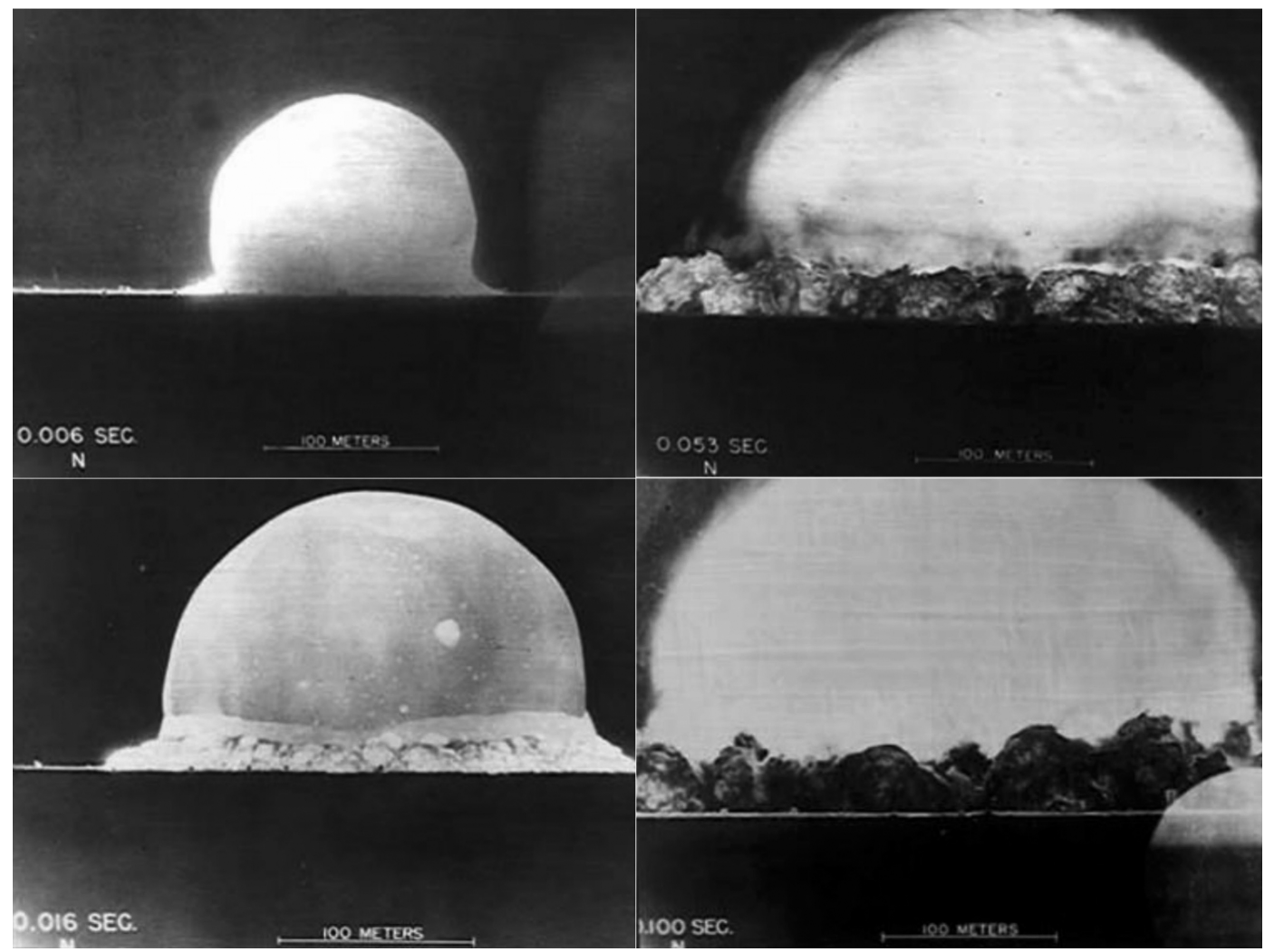

Figura 2: Frentes de choque em explosões de bombas atômicas. Figura de [3].

são: o número $N$ de remadores, que é, claramente, adimensional, a área do barco submersa em baixo da água $A$ (com dimensões de $L^{2}$ ) e a velocidade $v$ do barco, sendo a densidade da água irrelevante. A força de arrasto experimentada pelo barco é proporcional à velocidade ao quadradd

$$
F_{\text {arrasto }} \sim v^{2} A
$$

e a potência necessária para compensar a perda de energia é dada por

$$
P=F_{\text {arrasto }} v \sim v^{3} A .
$$

$\mathrm{O}$ volume submerso pode ser estimado como sendo linear no número de remadores, $V \sim N$, implicando que $A \sim N^{2 / 3}$. Assumimos também que todos os

\footnotetext{
${ }^{7} \mathrm{O}$ símbolo $\sim$ significa que não estamos interessados na dependência precisa em todas as grandeza do problema (e nem em constantes númericas adimensionais), mas somente em algumas grandezas selecionadas, como $v$ e $A$. Em particular, uma relação indicada com $\sim$ pode até ser inconsistente do ponto de vista dimensional, como, por exemplo, mais em baixo quando escrevemos $P \sim N$ : aqui estamos suprimindo a potência individual de cada remador, que deixaria a relação consistente. Em um certo sentido, este exemplo tem mais a ver com obter relações de "scaling" que com a análise dimensional.
}

remadores remem com a mesma potência, $P \sim N$. Juntando todas essas fórmulas, vemos que

$$
v \sim N^{1 / 9}
$$

e, consequentemente, o tempo para cobrir uma distância fixa vai como

$$
t \sim N^{-1 / 9}
$$

É interessante observar que essa predição da análise dimensional foi verificada [5] usando os tempos dos recordes olímpicos nas especialidades de remos e a predição é consistente com esses tempos com um erro de \pm 1.5 segundos!

\section{Unidades de medida}

O leitor atento deve ter reparado que, até esse momento, tomei muito cuidado em nunca usar a expressão unidades de medida, somente usei o termo "dimensões". A razão é que as duas coisas são muito diferentes: as dimensões são propriedades intrínsecas das grandezas físicas, enquanto as unidades de medida são convenções usadas para descrever dimensões (da mesma forma que um objeto é uma 
coisa diferente da palavra usada para descrevê-lo). Por exemplo, o metro é uma unidade para medir um comprimento $L$, não o comprimento em si.

A introdução de unidades de medida é necessária para efetuar medidas, que são obtidas comparando uma certa grandeza física com uma grandeza de referência cujas dimensões são as mesmas, especificando qual a grandeza de referência, como segue:

$$
\text { medida }=\frac{\text { grandeza física }}{\text { grandeza de referência }} \text { unidade }
$$

Não irei discutir aqui os vários sistemas de unidades de medida e nem a metrologia, que são assunto padrão nos livros-texto, como por exemplo [1.

O ponto principal da discussão que segue é, ao invés, ressaltar como esses sistemas de unidades são convencionais, dependendo de definições derivadas da nossa experiência local do universo. Por exemplo, o metro foi definido originalmente como uma certa fração do comprimento dos meridianos terrestres e, embora essa definição tenha sido refinada posteriormente, fica claro que é de natureza arbitrária. Na Figura 3, são representadas as unidades de massa, comprimento e volume que, atualmente, encontramse no museu de Sèvres.

É claro que, embora seja útil introduzir essas convenções para fins práticos, a física não pode depender delas, mas deve ser universal! Em particular, a física deve ser descrita e comunicada sem fazer referência a convenções locais. Essa observação nos motiva, portanto, a procurar sistemas de unidades de medida que também sejam universais e ultrapassem os confins das nossas experiências locais.
Uma estratégia para achar uma nova base de dimensões que seja universal é tentar utilizar leis universais da física, em particular usando as chamadas constantes fundamentais que aparecem nessas leis.

\subsection{As três constantes fundamentais da Na- tureza}

Há três constantes fundamentai: 8 na Natureza: a constante de Newton (G), a velocidade da luz (c) e a constante de Planck $(\hbar)$.

A constante de Newton entra ubiquamente nas leis da gravitação, descrevendo o acoplamento do campo gravitacional com a matéria. Por exemplo, entra na lei de gravitação de Newton

$$
F_{g}=G \frac{m_{1} m_{2}}{r^{2}}
$$

e também nas equações de Einstein

$$
R_{\mu \nu}-\frac{1}{2} R g_{\mu \nu}=\frac{8 \pi G}{c^{4}} T_{\mu \nu}
$$

Uma das principais características da gravidade é o fato de ser universal, ou seja, de afetar todas as coisas da mesma maneira. Isso se deve, justamente,

\footnotetext{
${ }^{8} \mathrm{O}$ leitor é convidado a ler um divertido debate sobre esse ponto entre três físicos famosos, cada um com um ponto de vista diferente sobre o assunto 6]. Nessa revisão, apresentamos, por razões pedagógicas, o ponto de vista mais comum, das três constantes fundamentais, que, naquela referência, é defendido pelo L. B. Okun. Foi também proposto em 7 que seria possível medir massas em termos de comprimentos e tempos. Isso rebaixaria a constante de Newton a ser um simples fator de conversão, deixando somente duas grandezas fundamentais, $c$ e $\hbar$.
}

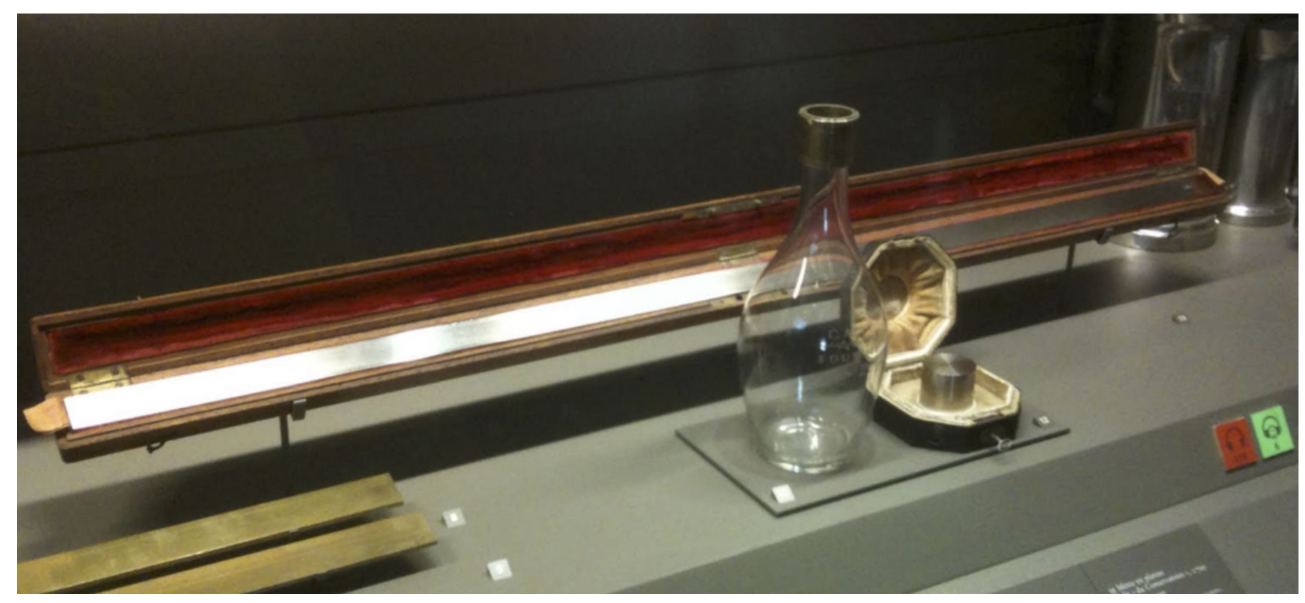

Figura 3: Unidades de medida para massa, comprimento e volume no Escritório Internacional de Pesos e Medidas de Sèvres, na França. Figura da Internet. 
ao fato de $G$ ser constante e igual para todas as coisas (campos, matéria, partículas, etc.).

A velocidade da luz é a velocidade com a qual a luz viaja no vácuo e representa a velocidade limite para qualquer objeto no universo, além de ser o fator de conversão entre enegia e massa, conforme a famosa equação de Einstein $E=m c^{2}$.

A constante de Planck representa a unidade mínima (ou quantum) de momento angular na mecânica quântica e entra em muitas equações, como na radiação de corpo negro ou nos níveis energéticos de átomos. Por exemplo, os níveis do átomo de hidrogênio são discretos e dados por

$$
E_{n}=-\frac{m_{\mathrm{e}} e^{4}}{2 \hbar^{2} n^{2}}, \quad n=1,2, \ldots
$$

O leitor pode encontrar mais detalhes sobre essas três constantes fundamentais nos livros-texto, como o [1. Nas unidades de medida do Sistema Internacional, essas três constantes têm valores numéricos dados por

$$
\begin{aligned}
& G=6.674 \times 10^{-11} \frac{\mathrm{m}^{3}}{\mathrm{~kg} \cdot \mathrm{s}^{2}}, \\
& c=2.998 \times 10^{8} \frac{\mathrm{m}}{\mathrm{s}}, \\
& \hbar=1.055 \times 10^{-34} \frac{\mathrm{m}^{2} \cdot \mathrm{kg}}{\mathrm{s}} .
\end{aligned}
$$

Daqui a pouco, precisaremos desses valores explícitos.

\section{Exemplo 6: Raio de Bohr}

Tendo mencionado a constante de Planck, podemos agora considerar uma aplicação muito importante da análise dimensional em mecânica quântica: queremos estimar qual é o tamanho típico de um átomo (de hidrogênio, por simplicidade), mas sem resolver a equação de Schrödinger para o sistema. A primeira coisa a ser feita é claramente identificar as grandezas físicas relevantes. Um átomo de hidrogênio é formado por um elétron, de massa $m_{\mathrm{e}}$ e carga elétrica $-e$, e por um próton, de massa $m_{\mathrm{p}}$ e carga $+e$. Parece, então, que temos que considerar as seguintes grandezas: $\hbar$ (claro, sendo isso um problema de mecânica quântica), o tamanho do átomo que queremos achar e que chamamos $a_{0}$, a carga $e$ e as duas massas $m_{\mathrm{e}}$ e $m_{\mathrm{p}}$. Uma observação crucial é que, na verdade, a massa que entra nessa conta deveria ser outra, a chamada massa reduzidd 9

$$
\mu=\frac{m_{\mathrm{e}} m_{\mathrm{p}}}{m_{\mathrm{e}}+m_{\mathrm{p}}} .
$$

Sendo o próton muito mais pesado que o elétron, podemos aproximar $\mu \simeq m_{\mathrm{e}}$. Portanto, a massa do próton não é de verdade relevante, pelo menos em uma primeira estimativa. ${ }^{10}$ A combinação de $\hbar, e$ e $m_{\mathrm{e}}$ com dimensões de comprimento resulta ser

$$
a_{0}=\frac{\hbar^{2}}{m_{\mathrm{e}} e^{2}},
$$

como pode ser visto usando o procedimento ilustrado na Seção 2. Esse comprimento é chamado de raio de Bohr e, de fato, é a quantidade que define o tamanho típico dos orbitais eletrônicos, como pode ser visto explicitamente resolvendo a equação de Schrödinger. Usando os valores explícitos no Sistema Internacional, resulta que $a_{0} \simeq 5.29 \times 10^{-11} \mathrm{~m}$.

\subsection{O cubo da física}

É muito interessante observar que as três constantes introduzidas acima oferecem um jeito muito legal de organizar toda a física em um diagrama chamado de cubo da física ${ }^{11}$ Ver a Figura 4 .

Consideremos, inicialmente, o caso em que não haja essas constantes, ou seja " $G \rightarrow 0, \hbar \rightarrow 0$, $c \rightarrow \infty " 12$ Esse é o caso da mecânica de Newton sem a gravidade. "Ligando" somente $G$, achamos a gravitação de Newton; ligando somente $c$, achamos a relatividade restrita; ligando somente $\hbar$, achamos a mecânica quântica.

\footnotetext{
${ }^{9}$ Quando um problema depende somente da distância relativa entre dois objetos, é sempre útil trocar aqueles dois objetos (de massa $m_{1}$ e $m_{2}$ e coordenadas $\vec{x}_{1}$ e $\vec{x}_{2}$ ) por dois "objetos virtuais", um com massa reduzida $\mu=m_{1} m_{2} /\left(m_{1}+m_{2}\right)$ e coordenada $\vec{r}=\vec{x}_{1}-\vec{x}_{2}$, chamada de relativa, e um outro de massa igual à soma $M=m_{1}+m_{2}$ das massas e coordenada $\vec{R}=\left(m_{1} \vec{r}_{1}+m_{2} \vec{r}_{2}\right) / M$, chamada de centro de massa. Toda a dinâmica do problema está contida no objeto com coordenada relativa, enquanto o objeto no centro de massa não exerce nenhum papel interessante.

${ }^{10}$ Refinando as coisas, podemos pensar que $a_{0}$ depende também de uma função $f\left(m_{\mathrm{e}} / m_{\mathrm{p}}\right)$ da razão das massas, que, porém, não pode ser determinada pela análise dimensional, por ser adimensional.

${ }^{11} \mathrm{Ou}$ cubo de Bronshtein-Zelmanov-Okun, ver por exemplo 8 . ${ }^{12}$ Como deve ter ficado claro pela discussão anterior, isso não é estritamente correto, pois $G, c$ e $\hbar$ são dimensionais, enquanto 0 ou $\infty$ são adimensionais. O limite significa que $G$ e $\hbar$ são muito menores que qualquer outra grandeza com as mesmas dimensões no problema (e é a razão pela qual coloquei as aspas) e que $c$ é muito maior de qualquer outra velocidade no problema.
} 


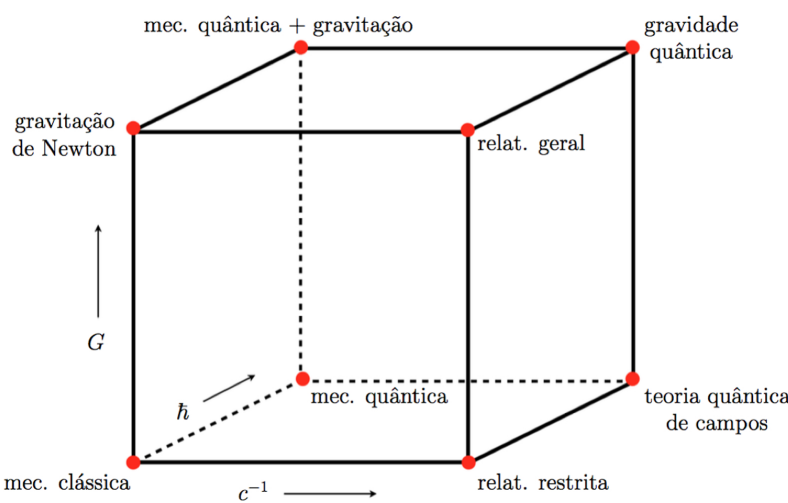

Figura 4: $\mathrm{O}$ cubo da física [8].

Podemos agora ligar dois constantes ao mesmo tempo. Ligando $G$ e $c$, achamos a relatividade geral de Einstein; ligando $G$ e $\hbar$, achamos a mecânica quântica não relativística mais gravitação; ligando $c$ e $\hbar$, achamos a teoria quântica de campos. O último desafio da física teórica, o Santo Graal, é entender o caso quando todas as três constantes são ligadas, ou seja a gravidade quântica, que é o casamento entre mecânica quântica e relatividade geral.

\subsection{O cerne da teoria quântica de campos}

Motivados pela aparição da teoria quântica de campos no cubo da física, vamos gastar duas palavras para esboçar a ideia principal dessa teoria. A união entre a relatividade restrita e a mecânica quântica tem uma consequência crucial: partículas podem nascer e morrer. Em outras palavras, o número de partículas no problema não é constante, ou conservado, como acontece na mecânica clássica ou na mecânica quântica sem relatividade restrita.

$\mathrm{Na}$ relatividade restrita, a energia é equivalente à massa, conforme a fórmula mencionada acima: $E=m c^{2}$. Tendo energia, podemos criar massa, ou seja, podemos criar partículas. Na teoria quântica de campos, o vácuo, que é o estado de menor energia, não é vazio, mas repleto de partículas e antipartículas virtuais. Podemos excitar essas partículas e anti-partículas virtuais e convertê-las em partículas e anti-partículas reais, tendo energia a disposição, por exemplo, usando aceleradores como o LHC. Também é possível, porém, que pares de partículas e anti-partículas ${ }^{13}$ surjam do vácuo espontaneamente

\footnotetext{
${ }^{13} \overline{\text { Devem ser pares de }}$ partículas e correspondentes antipartículas (partículas de carga oposta) para respeitar critérios de conservação, como a conservação da carga elétrica, que no vácuo é zero.
}

para, logo depois, se aniquilarem, ver a Figura 5 (Esq.).

Claramente, isso seria uma violação da conservação da energia, pois o vácuo tem energia zero, enquanto o par tem energia igual, pelo menos, à massa deste, que é duas vezes a massa da partícula, pois partículas e correspondentes anti-partículas têm a mesma massa. Ver a Figura 5 (Dir.). A resolução desse paradoxo está na mecânica quântica, em particular, na relação de indeterminação de Heisenberg

$$
\Delta E \Delta t \geq \frac{\hbar}{2}
$$

que não nos permite medir a energia com precisão arbitrária em intervalos finitos de tempo. Podemos tolerar uma violação da conservação da energia igual a $\Delta E$ para um (curto) intervalo de tempo $\Delta t \leq$ $\frac{\hbar}{2 \Delta E}$, que é justamente o intervalo de tempo que a partícula e a anti-partícula vivem antes de se aniquilarem uma com a outra.
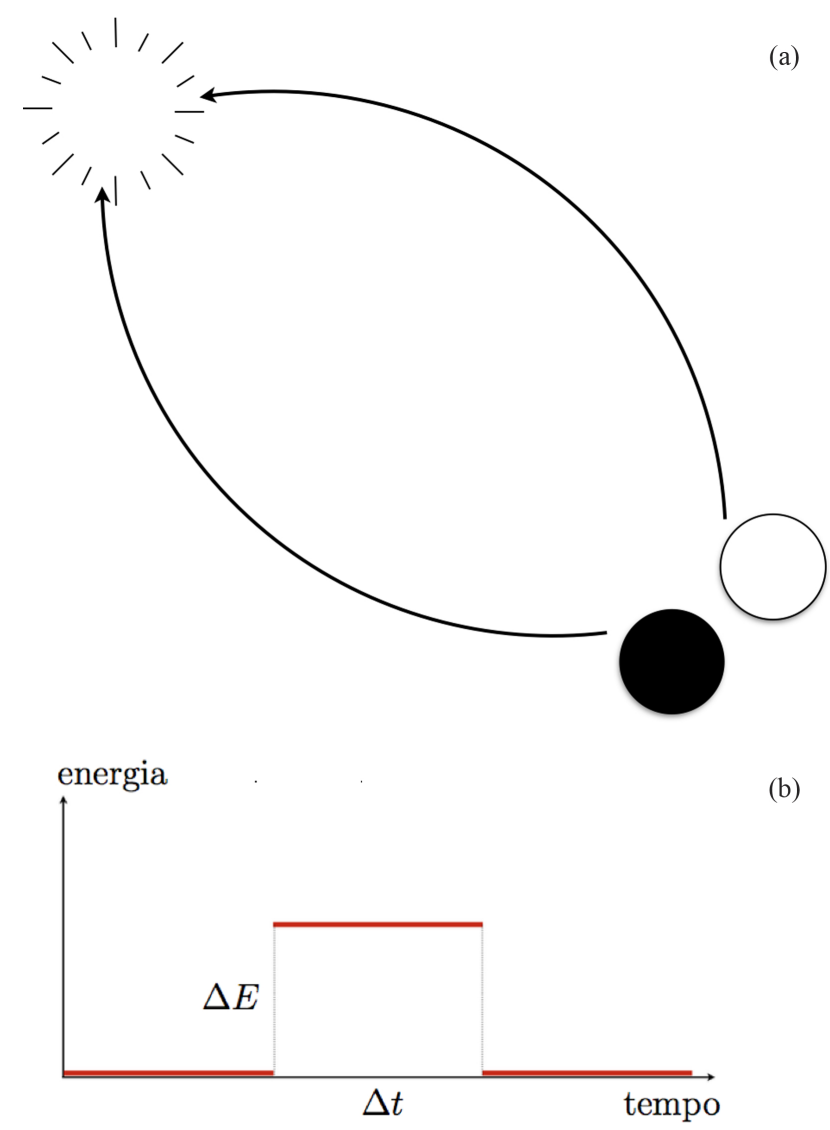

(b)

Figura 5: (a) Produção e aniquilação no vácuo de um par partícula/anti-partícula. (b) A conservação de energia pode ser "violada" durante breves intervalos de tempo, graças ao princípio de indeterminação entre energia e tempo. 


\section{Exemplo 7: Constante de estrutura fina}

Consideremos, agora, elétrons em teoria quântica de campos. As grandezas dimensionais à nossa disposição são: a constante de Planck $\hbar$, a velocidade da luz $c$, a carga do elétron $e$ e a massa do elétron $m_{\mathrm{e}}$. A pergunta óbvia é a seguinte: podemos construir uma combinação adimensional $Y$ dessas grandezas? Para fazer isso, escrevemos

$$
\begin{aligned}
& Y=\hbar^{a_{1}} c^{a_{2}} e^{a_{3}} m_{\mathrm{e}}^{a_{4}} \quad \rightarrow[Y]=1= \\
& M^{a_{1}+\frac{1}{2} a_{3}+a_{4}} L^{2 a_{1}+a_{2}+\frac{3}{2} a_{3}} T^{-a_{1}-a_{2}-a_{3}},
\end{aligned}
$$

onde usamos que a carga elétrica tem dimensões de $\sqrt{M L^{3} T^{-2}}$. Uma solução é $\left(a_{1}=-1, a_{2}=-1, a_{3}=\right.$ $\left.2, a_{4}=0\right)$ e a correspondente combinação adimensional é dada por

$$
\frac{e^{2}}{\hbar c}=7.297 \times 10^{-3} \simeq \frac{1}{137} .
$$

A observação importante é que esse é um número pequeno. O leitor deve lembrar agora a terceira observação na Seção 2 números pequenos e adimensionais podem ser usados como parâmetros nas expansões. De fato, o número acima é chamado constante de estrutura find ${ }^{14}$ e aparece nas expansões perturbativas de algumas teorias de campos, como a eletrodinâmica quântica (QED).

\section{Sistema de unidades naturais}

O leitor atento deve ter observado que as três constantes introduzidas acima têm dimensões independentes:

$$
\begin{aligned}
& {[G]=M^{-1} L^{3} T^{-2}} \\
& {[c]=L T^{-1}} \\
& {[\hbar]=M L^{2} T^{-1} .}
\end{aligned}
$$

É possível, portanto, usá-las para definir uma nova base de dimensões, as dimensões de $[G],[c]$ e $[\hbar]$. Daqui, podemos mudar de base de novo e voltar a uma base de $M, L$ e $T$. Explicitamente, vamos escrever

$$
[G]^{a_{1}}[c]^{a_{2}}[\hbar]^{a_{3}}=M^{-a_{1}+a_{3}} L^{3 a_{1}+a_{2}+2 a_{3}} T^{-2 a_{1}-a_{2}-a_{3}} .
$$

Agora, queremos achar combinações com dimensões de massa, comprimento e tempo:

$$
\begin{gathered}
\text { massa } \\
-a_{1}+a_{3}=1 \\
3 a_{1}+a_{2}+2 a_{3}=0 \\
-2 a_{1}-a_{2}-a_{3}=0
\end{gathered}
$$

$$
\begin{gathered}
\text { comprimento } \\
-a_{1}+a_{3}=0 \\
3 a_{1}+a_{2}+2 a_{3}=1 \\
-2 a_{1}-a_{2}-a_{3}=0
\end{gathered}
$$

$$
\begin{gathered}
\text { tempo } \\
-a_{1}+a_{3}=0 \\
3 a_{1}+a_{2}+2 a_{3}=0 \\
-2 a_{1}-a_{2}-a_{3}=1 .
\end{gathered}
$$

As soluções são chamadas massa de Planck $m_{\mathrm{P}}$, comprimento de Planck $\ell_{\mathrm{P}}$ e tempo de Planck $t_{\mathrm{P}}$ :

$$
m_{\mathrm{P}}=\sqrt{\frac{\hbar c}{G}} \ell_{\mathrm{P}}=\sqrt{\frac{G \hbar}{c^{3}}} t_{\mathrm{P}}=\sqrt{\frac{G \hbar}{c^{5}}} .
$$

O ponto crucial desse exercício é que a massa, o comprimento e o tempo de Planck têm um caráter universal, pois foram definidas a partir de constantes fundamentais! Eles representam as escalas de massa (de objetos elementares), comprimento e tempo onde efeitos de gravidade quântica viram importantes. Em outras palavras, quando consideremos fenômenos que acontecem em comprimentos de Planck, ou em escalas de tempo de Planck, o nosso entendimento

\footnotetext{
${ }^{14} \mathrm{Na}$ verdade, não é constante e depende da energia do sistema, mas isso é uma outra história!
}

usual de espaço-tempo da relatividade geral deve ser modificado, para incluir efeitos quânticos. Isso tem a ver com o último vértice do cubo da física, na Figura 4.

Os valores dessas quantidades no Sistema Internacional são dados por

$$
\begin{aligned}
& m_{\mathrm{P}}=2.176 \times 10^{-5} \mathrm{~g}, \\
& \ell_{\mathrm{P}}=1.616 \times 10^{-33} \mathrm{~cm}, \\
& t_{\mathrm{P}}=5.391 \times 10^{-44} \mathrm{~s} .
\end{aligned}
$$

Reparamos que esses valores são extremos! O comprimento de Planck e o tempo de Planck são extremamente menores que todos os comprimentos e tempos aos quais estamos acostumados. A massa de Planck é, por outro lado, muito maior que a massa 
das partículas elementares (por exemplo, é 19 ordens de magnitude maior que a massa do próton) 15

Agora, é natural ir um passo adiante e definir um novo sistema de unidades, chamado de unidades naturais ou unidades de Planck. Nesse sistema declaramos que

$$
c \rightarrow 1, \quad \hbar \rightarrow 1, \quad G \rightarrow 1
$$

Vamos entender o que isso significa. Declarar que a velocidade da luz tende a 1 , ou seja, um parâmetro adimensional, implica em comprimentos e tempos com a mesma dimensão. Isso deve ser familiar para o leitor: um ano-luz é uma medida de comprimento, embora a palavra "ano" seja usada na expressão, e é a distância que a luz viaja em um ano $\left(\simeq 9.46 \times 10^{12}\right.$ $\mathrm{km})$. Velocidades são, portanto, medidas em relação à velocidade da luz e variam de 0 a 1 , que é a velocidade limite. Outra consequência é que $E=$ $m c^{2}$ vira $E=m$, ou seja, energia e massa vão também ter as mesmas dimensões.

Quando $c \rightarrow 1$, a constante de Planck $\hbar$ vai ter dimensões de $M L$. Declarar que a mesma $\hbar$ vira 1 significa portanto que massas (e energias) viram inversos de comprimentos. Por fim, declarar que $G$ vira 1 significa que a massa ou o comprimento de referência são 1.

Notamos que, na prática, fala-se que $c=1, \hbar=1$ e $G=1$, em vez de colocar o símbolo de limite, mas o significado é o mesmo. Vale a pena ressaltar um ponto que, às vezes, confunde os alunos: colocar $c=1, \hbar=1$ e $G=1$ não significa "perder informações" sobre as grandezas e dimensões do problema! Querendo, podemos sempre inserir univocamente de volta $c, \hbar$ e $G$ nas fórmulas, somente usando a análise dimensional.

\section{Exemplo 8: Radiação Hawking e entropia de buracos negros}

Buracos negros estão entre os objetos mais interessantes e misteriosos do universo. Os buracos negros mais simples, presentes na relatividade geral, são chamados de Schwarzschild16 São estáticos e esféricos com um raio chamado raio de Schwarzs-

\footnotetext{
${ }^{15} \mathrm{O}$ ponto aqui é que devemos comparar a massa de Planck, que é algo fundamental, com a massa de coisas também fundamentais, ou elementares, e não compostas. Coisas compostas podem claramente ter massas bem maiores que $m_{\mathrm{P}}$ !

${ }^{16}$ Os buracos negros astrofísicos não são de Schwarzschild, pois geralmente têm momento angular e rodam.
}

child e dado por

$$
R_{\mathrm{S}}=\frac{2 G M}{c^{2}},
$$

onde $M$ é a massa do buraco negro. Para ter uma ideia, um buraco negro com massa igual à massa do Sol tem raio $R_{\mathrm{S}} \simeq 3 \mathrm{~km}$. O leitor deve ter ouvido falar que buracos negros são tão densos que deformam o espaço-tempo ao seu redor, ao ponto de não deixar escapar nada, nem a luz, que entra no raio de Schwarzschild (onde há o chamado horizonte de eventos). De fato, isso é verdade somente se desprezarmos efeitos quânticos. Bekenstein e Hawking demonstraram, nos anos '70, que buracos negros emitem uma radiação, e portanto têm propriedades termodinâmicas, como temperatura e entropia. Para mais detalhes, ver, por exemplo, o ótimo livrotexto 9 .

Uma maneira heurística de entender a fonte dessa radiação (chamada de radiação Hawking) é lembrar a história das partículas da teoria de campos que surgem do vácuo e se aniquilam. Se o horizonte de eventos se encontra exatamente entre a partícula e a anti-partícula que surgiram do vácuo, a partícula dentro do horizonte será absorvida pelo buraco negro, enquanto a partícula fora do horizonte deixa de ser virtual para virar real e se propagar como uma partícula ordinária (pois ela não tem mais um parceiro para se aniquilar). Ver a Figura 6. A radiação Hawking pode então ser pensada como se fosse constituída por essas partículas que, de virtuais, viraram reais.

É possível estimar a temperatura e entropia do buraco negro usando a análise dimensional [9]. Adotamos um sistema de unidades $\operatorname{com} c=1=\hbar$, mas $G \neq 1$. Os parâmetros do problema são, portanto, $G$ e $M$ (e $R_{\mathrm{S}}$ que, porém, depende desses dois). Nessas unidades, a combinação $G M$ tem dimensões de comprimento ou de inverso de massa. Lembrando que temperatura é energia (a energia média dos graus de libertade microscópicos), a temperatura do buraco negro deve ser dada por

$$
T \sim \frac{1}{G M} .
$$

Fazendo a conta detalhada e colocando todos os fatores, dimensionais e adimensionais, seria $T=$ $\hbar c^{3} / 8 \pi G M$. Vemos, portanto, que: 1$)$ essa radiação é, de fato, um efeito quântico, pois se anula quando $\hbar$ é desprezível; 2) é explosiva para massas pequenas! É interessante frisar que, para um buraco negro com 


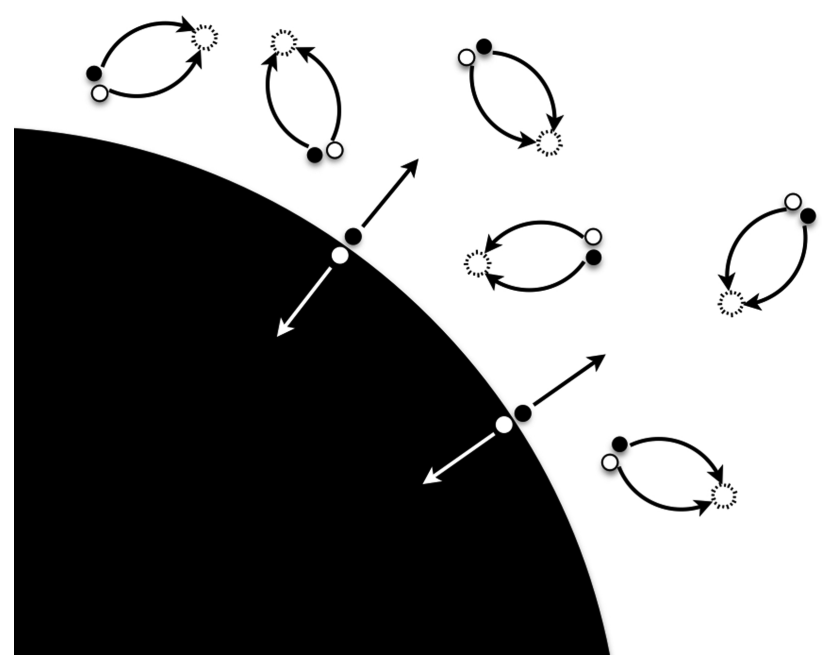

Figura 6: A radiação Hawking de um buraco negro. As partículas virtuais que nascem perto do horizonte de eventos são separadas das próprias parceiras e viram reais.

massa solar, a temperatura é muito baixa: $T \simeq 10^{-7}$ $\mathrm{K}$.

Conforme a primeira lei da termodinâmica, $d E=$ $T d S$, associada a uma temperatura há uma entropia. Lembrando que a massa do buraco negro é a energia (pois $c=1$ ), podemos integrar essa lei usando (38)

$$
\frac{d E}{d S}=\frac{d M}{d S} \sim \frac{1}{G M} \quad \rightarrow \quad S \sim G M^{2}
$$

Para uma massa solar, isso dá uma entropia enorme: $S \simeq 10^{77}$. Em termos do raio de Schwarzschild, podemos re-expressar a entropia como

$$
S \sim \frac{R_{\mathrm{S}}^{2}}{G}
$$

ou seja, a entropia $S$ de um buraco negro é proporcional à área do horizonte, que é uma esfera, $A=4 \pi R_{\mathrm{S}}^{2}{ }^{17}$ Isso é muito diferente do que acontece em sistemas termodinâmicos usuais, onde a entropia é extensiva e varia com o volume da área ocupada, e não com a área da borda desse volume! Isso tem consequências muito profundas. As mais importantes são, talvez, o princípio holográfico 10 e a correspondência AdS/CFT [11].

Lembrando a discussão acima sobre a criação de partículas em aceleradores, podemos, agora, dar uma interpretação muito interessante ao comprimento de Planck como comprimento mínimo. A ideia é que, em um mundo sem gravidade, podemos, em

${ }^{17}$ Colocando todos os fatores, teríamos $S=k_{\mathrm{B}} c^{3} A / 4 G$, com $k_{\mathrm{B}}$ a constante de Boltzmann. princípio, sondar distâncias arbitrariamente pequenas aumentando a energia do acelerador. Conforme a mecânica quântica, com energia $E$ do feixe podemos sondar partículas localizadas em uma região chamada comprimento de de Broglie, dada por

$$
\ell_{\mathrm{dB}} \sim \frac{1}{E}
$$

em unidades de $c=\hbar=1$. Introduzindo a gravidade, porém, o cenário muda drasticamente, pois há buracos negros. Uma energia $E$ concentrada dentro de um raio de Schwarzschild (37) com $M \sim E$ vai, de fato, colapsar em um buraco negro. O feixe do acelerador vai formar um buraco negro quando

$$
R_{\mathrm{S}} \sim G E \gtrsim \ell_{\mathrm{dB}} \sim \frac{1}{E} \rightarrow E \gtrsim \sqrt{\frac{1}{G}}=m_{\mathrm{P}},
$$

sempre em unidades de $c=1=\hbar$. Portanto, distâncias menores que $\ell_{\mathrm{P}}$ não são acessíveis, pois um buraco negro de $R_{\mathrm{S}} \gtrsim \ell_{\mathrm{P}}$ é formado no processo e não podemos olhar dentro de um buraco negro!

Vamos terminar essa seção com um simples exercício [12]. A ideia é se convencer que efeitos de gravidade quântica são desprezíveis em situações ordinárias. Já vimos, por exemplo, que a temperatura da radiação Hawking é extremamente baixa para buracos negros com massas comparáveis à do Sol. Alguns buracos negros astrofísicos chegam a ter mais que $10^{10}$ massas solares. Para eles, essa temperatura seria ainda menor, pois varia com o inverso da massa.

Agora queremos estimar a importância da interação gravitacional em átomos. Qual seria o raio de Bohr do átomo de hidrogênio se a interação entre elétron e próton fosse somente gravitacional e não eletromagnética? Para resolver isso, lembramos que,

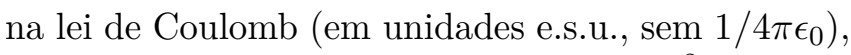
a intensidade da interação varia com $-e^{2}$, ou seja, o produto das cargas de elétron e próton. Por outro lado, na lei de gravitação de Newton, o acoplamento gravitacional varia com $G$ vezes o produto das massas: $G m_{\mathrm{e}} m_{\mathrm{p}}$. Portanto, para achar o "raio de Bohr gravitacional" devemos trocar esses acoplamentos em (27):

$$
\begin{aligned}
& a_{0}=\frac{\hbar^{2}}{m_{\mathrm{e}} e^{2}} \rightarrow a_{0}^{(g)}=\frac{\hbar^{2}}{m_{\mathrm{e}}\left(G m_{\mathrm{e}} m_{\mathrm{p}}\right)} \simeq \\
& 1.20 \times 10^{31} \mathrm{~cm} .
\end{aligned}
$$

A interação seria tão pequena que o raio do átomo resultaria ser maior que o universo observável! Isso 
é uma consequência direta do fato de $G$ ser tão pequena.

Efeitos de gravidade quântica devem ser incluídos em situações extremas, como perto das singularidades no centro de buracos negros ou no estudo do universo primordial, quando temperatura e densidade eram altíssimas, da ordem da escala de Planck.

\section{Conclusão}

Espero ter convencido o leitor sobre o poder e a elegância da análise dimensional: em muitas situações, podemos ter indicações sobre quais são as escalas e as combinações de parâmetros relevantes em um problema, simplesmente analisando as grandezas dimensionais do mesmo. Vimos isso em vários exemplos de várias áreas da física, da mecânica básica até a efeitos quânticos na gravidade.

Agora, às vezes, a análise dimensional e as expectativas baseadas em comparações com escalas naturais em um problema fracassam tragicamente: é o caso dos chamados problemas de hierarquias. Há muitas hierarquias não ainda explicadas e que representam algumas das perguntas em aberto mais importantes da física. Por exemplo, vimos que a massa do próton é muito menor da massa de Planck. Por quê? O exemplo arquetípico desses problemas é o problema da constante cosmológica responsável pela expansão acelerada do universo: a discrepância entre expectativa e medição é de 120 ordens de magnitude naquele caso!

A resposta a qualquer uma dessas perguntas seria, com certeza, digna de um prêmio Nobel. Vamos deixar uma discussão mais aprofundada sobre esses temas para uma outra ocasião.

\section{Agradecimentos}

Agradeço a Prof.a Renata Zukanovich Funchal e toda a organização do Convite à Física do Instituto de Física da USP, pelo convite a apresentar esse material em uma palestra em agosto de 2015. Agradeço também a Deborah Liguori para a ajuda com a revisão do texto. A minha pesquisa é financiada em parte pelo CNPq e em parte pela FAPESP (projetos 2014/18634-9 e 2015/17885-0). Comentários e pedidos de esclarecimentos são muito bem vindos.

\section{Referências}

[1] M.H. Nussenzveig, Curso de Física Básica (Editora Edgard Blücher, São Paulo, 2008).

[2] E. Buckingham, Phys. Rev. 4, 345 (1914).

[3] http://www.atmosp.physics.utoronto. $\mathrm{ca} / \mathrm{people/codoban/PHY138/Mechanics/}$ dimensional.pdf.

[4] T. McMahon, Science 173, 349 (1971).

[5] Www.sciencebits.com/rowers

[6] M.J. Duff, L.B. Okun and G. Veneziano, JHEP 0203, 023 (2002).

[7] G.E.A. Matsas, V. Pleitez, A. Saa and D.A.T. Vanzella, disponível em arXiv:0711.4276.

[8] L.B. Okun, in: Multiple Facets of Quantization and Supersymmetry, edited by M. Olshanetsky and A. Vainshtein (World Scientific, Singapura, 2002), p. 670-675, disponível em hep-ph/0112339

[9] A. Zee, Einstein Gravity in a Nutshell (Princeton Univ. Press, Princeton, 2013).

[10] D. Bigatti and L. Susskind, disponível em hep-th/ 0002044

[11] J.M. Maldacena, Int. J. Theor. Phys. 38, 1113 (1999). Adv. Theor. Math. Phys. 2, 231 (1998), disponível em hep-th/9711200.

[12] B. Zwiebach, A First Course in String Theory (Cambridge Univ. Press, Cambridge, 2004). 